\title{
Universality of One-Dimensional Reversible and Number-Conserving Cellular Automata
}

\author{
Kenichi Morita \\ Department of Information Engineering, Hiroshima University \\ Higashi-Hiroshima, 739-8527, Japan \\ km@hiroshima-u.ac.jp
}

\begin{abstract}
We study one-dimensional reversible and number-conserving cellular automata (RNCCA) that have both properties of reversibility and number-conservation. In the case of 2-neighbor RNCCA, GarcíaRamos proved that every RNCCA shows trivial behavior in the sense that all the signals in the RNCCA do not interact each other. However, if we increase the neighborhood size, we can find many complex RNCCAs. Here, we show that for any one-dimensional 2-neighbor reversible partitioned CA (RPCA) with $s$ states, we can construct a 4-neighbor RNCCA with $4 s$ states that simulates the former. Since it is known that there is a computationally universal 24-state 2-neighbor RPCA, we obtain a universal 96-state 4-neighbor RNCCA.
\end{abstract}

\section{Introduction}

A reversible cellular automaton (RCA), and a number-conserving cellular automaton (NCCA) are kinds of abstract spatiotemporal models that reflect physical properties of reversibility, and conservation (of mass, energy, etc.), respectively. A reversible and number-conserving cellular automaton (RNCCA) is thus a model that has both these properties. Though an RNCCA is a very restricted subclass of a CA, its behavior can be complex if we increase the neighborhood size and the number of states.

So far, NCCAs have been extensively studied, and various properties and characterizations of them have been given [1, 3, 4, 5, 7, 9]. In [10] Moreira investigated universality and decidability of NCCAs. As for RNCCAs, Schranko and de Oliveira [15] made an experimental study on one-dimensional RNCCAs, and showed that an $s$-state $n$-neighbor RNCCA rule can be decomposed into $s$-state 2-neighbor RNCCA rules when $s$ and $n$ are very small. García-Ramos [6] proved that, in the 2-neighbor case (i.e., radius $1 / 2$ ), every RNCCA is a shift-identity product cellular automaton (SIPCA). An SIPCA is an RNCCA composed of "shift CAs" in which all signals are right-moving, and "identity CAs" in which all signals are stationary. Hence, in general, an SIPCA has both right-moving and stationary signals, but each signal is independent to others. Namely, every right-moving signal simply goes through stationary signals without affecting them. Therefore, all the 2-neighbor RNCCAs show trivial behaviors in the sense that the signals do not interact each other. On the other hand, Imai, Martin and Saito [8] showed that, in the 3neighbor case (i.e., radius 1), there are RNCCAs in which some signals can interact with others, and thus they show some nontrivial behavior. However, it is not known whether there exists a computationally universal 3-neighbor RNCCA.

In this paper, we investigate the 4-neighbor case (i.e., radius 3/2), and prove there is a computationally universal RNCCA. We show that for any given 2-neighbor $s$-state reversible partitioned CA (RPCA) we can construct a 4-neighbor $4 s$-state RNCCA that simulates the former. On the other hand, it is known that there is a computationally universal 2-neighbor 24-state RPCA [12], which can simulate any cyclic tag system proposed by Cook [2]. By this, we can obtain a universal 4-neighbor 96-state RNCCA.

E. Formenti (Ed.): AUTOMATA and JAC 2012 conferences EPTCS 90, 2012, pp. 142-150 doi 10.4204/EPTCS.90.12 (c) K. Morita

This work is licensed under the Creative Commons Attribution License. 
Computational universality in a variant of a one-dimensional RNCCA was studied by Morita and Imai [14], but this CA was not in the standard framework of NCCAs, because a partitioned CA (PCA) was used as an NCCA model. Though a reversible PCA is a subclass of a standard RCA, the numberconserving property of a PCA is somehow different from an NCCA, since each cell of a PCA has several parts. Namely, while each cell of a usual NCCA has a single number, that of a number-conserving PCA has a "tuple" of numbers. Therefore, this paper gives the first universality result of an RNCCA in the standard framework of an NCCA.

\section{Preliminaries}

A one-dimensional cellular automaton $(C A)$ is a system defined by

$$
A=(\mathbb{Z}, Q, N, f, \#) .
$$

Here, $\mathbb{Z}$ is the set of all integers where cells are placed. $Q$ is a non-empty finite set of states of each cell. $N$ is a neighborhood, which is an element of $\mathbb{Z}^{m}(m=1,2, \ldots)$. Hence $N$ can be written as $N=\left(n_{1}, \ldots, n_{m}\right)$ where $n_{i} \in \mathbb{Z}(i \in\{1, \ldots, m\}), f: Q^{m} \rightarrow Q$ is a local function that determines a state transition of each cell depending on the states of its $m$ neighboring cells. \# $\in Q$ is a quiescent state that satisfies $f(\#, \ldots, \#)=\#$. If $f\left(q_{1}, \ldots, q_{m}\right)=q$ holds for $q_{1}, \ldots, q_{m}, q \in Q$, we call this relation $f\left(q_{1}, \ldots, q_{m}\right)=q$ a transition rule of $A$. Thus $f$ can be described as a finite set of transition rules.

A configuration over $Q$ is a mapping $\alpha: \mathbb{Z} \rightarrow Q$. Let $\operatorname{Conf}(Q)$ denote the set of all configurations over $Q$, i.e., $\operatorname{Conf}(Q)=\{\alpha \mid \alpha: \mathbb{Z} \rightarrow Q\}$. A configuration $\alpha$ is called finite if the set $\{x \mid x \in \mathbb{Z} \wedge \alpha(x) \neq \#\}$ is finite. Otherwise it is infinite. The set of all finite configurations is denoted by $\operatorname{Conf}_{\text {fin }}(Q)$. Applying the local function $f$ to all the cells in $\mathbb{Z}$ simultaneously, we can obtain a global function $F$ of $A$ that determines how a configuration changes to another. More precisely, $F: \operatorname{Conf}(Q) \rightarrow \operatorname{Conf}(Q)$ is defined by the following formula.

$$
\forall \alpha \in \operatorname{Conf}(Q), x \in \mathbb{Z}: F(\alpha)(x)=f\left(\alpha\left(x+n_{1}\right), \ldots, \alpha\left(x+n_{m}\right)\right)
$$

It means that the next state of a cell at the position $x$ is determined by the present states of $m$ cells at the positions $x+n_{1}, \ldots, x+n_{m}$ using the local function $f$. If $N=(-r,-r+1, \ldots, 0, \ldots, r-1, r)$ for some natural number $r \in \mathbb{N}$, then $A$ is called a CA of radius $r$. If $N=(-r,-r+1, \ldots, 0, \ldots, r-2, r-1)$ for some positive integer $r \in \mathbb{N}-\{0\}$, then $A$ is called a CA of radius $(2 r-1) / 2$. Hereafter, we call a CA of radius $r((2 r-1) / 2$, respectively) by a $(2 r+1)$-neighbor CA $(2 r$-neighbor $\mathrm{CA})$.

Let $A$ be a CA, and $F$ be its global function. $A$ is called a reversible $C A$ (RCA) iff $F$ is an injection, i.e., it satisfies the following condition.

$$
\forall \alpha_{1}, \alpha_{2} \in \operatorname{Conf}(Q): \alpha_{1} \neq \alpha_{2} \Rightarrow F\left(\alpha_{1}\right) \neq F\left(\alpha_{2}\right)
$$

A more detailed description on the definition of an RCA is found e.g. in [11].

A number-conserving CA is a one such that each cell's state is an integer, and their sum in a configuration is conserved throughout the evolving process. So far, several definitions and characterizations have been given for number-conserving CAs [1, 3, 4, 7]. Durand, Formenti and Róka [3] proved that the three notions, periodic-number-conserving, finite-number-conserving, and number-conserving (for infinite configurations), are all equivalent. In this paper, we employ the notion of finite-number-conserving to define a number-conserving $\mathrm{CA}$. 
Let $A=(\mathbb{Z}, Q, N, f, 0)$ be a CA, where $Q=\{0, \ldots, s-1\}(s \in \mathbb{N}-\{0\})$, and $F$ be its global function. The CA $A$ is called finite-number-conserving, if the following condition holds.

$$
\forall \alpha \in \operatorname{Conf}_{\text {fin }}(Q): \sum_{x \in \mathbb{Z}} \alpha(x)=\sum_{x \in \mathbb{Z}} F(\alpha)(x)
$$

A CA is called a number-conserving cellular automaton (NCCA), if it is finite-number-conserving.

A CA that satisfies both reversibility and finite-number-conserving conditions is called a reversible number-conserving CA (RNCCA).

Next, we give a definition of a partitioned CA, because, in the next section, we will show a method of converting a reversible partitioned CA into an RNCCA. A one-dimensional partitioned cellular automaton (PCA) is defined by

$$
P=\left(\mathbb{Z},\left(Q_{1}, \ldots, Q_{m}\right),\left(n_{1}, \ldots, n_{m}\right), f\right) .
$$

Here, $Q_{i}(i=1, \ldots, m)$ is a non-empty finite set of states of the $i$-th part of each cell, and thus the state set of each cell is $Q=Q_{1} \times \cdots \times Q_{m}$. The $m$-tuple $\left(n_{1}, \ldots, n_{m}\right) \in \mathbb{Z}^{m}$ is a neighborhood, and $f: Q \rightarrow Q$ is a local function.

Let $\operatorname{pr}_{i}: Q \rightarrow Q_{i}$ be the projection function such that $\operatorname{pr}_{i}\left(q_{1}, \ldots, q_{m}\right)=q_{i}$ for all $\left(q_{1}, \ldots, q_{m}\right) \in Q$. The global function $F: \operatorname{Conf}(Q) \rightarrow \operatorname{Conf}(Q)$ of $P$ is defined as the one that satisfies the following formula.

$$
\forall \alpha \in \operatorname{Conf}(Q), x \in \mathbb{Z}: F(\alpha)(x)=f\left(\operatorname{pr}_{1}\left(\alpha\left(x+n_{1}\right)\right), \ldots, \operatorname{pr}_{m}\left(\alpha\left(x+n_{m}\right)\right)\right)
$$

By above, one-dimensional PCA of radius $1 / 2$ is defined as follows.

$$
P=(\mathbb{Z},(C, R),(0,-1), f)
$$

Each cell has two parts, i.e., center and right parts, and their state sets are $C$ and $R$. The next state of a cell is determined by the present states of the center part of this cell, and the right part of the left-neighbor cell (not depending on the whole two parts of the two cells). Figure 1 shows its cellular space, and how the local function $f$ is applied. Note that, here, the neighborhood is $(0,-1)$ rather than $(-1,0)$.

It is easy to show the following lemma that states the equivalence of local and global injectivity of a PCA [13].

Lemma 1 Let $P=\left(\mathbb{Z},\left(Q_{1}, \ldots, Q_{m}\right),\left(n_{1}, \ldots, n_{m}\right), f\right)$ be a $P C A$, and $F$ be its global function. Then, the local function $f$ is injective, iff the global function $F$ is injective.

A PCA with an injective local function is thus called a reversible PCA (RPCA).

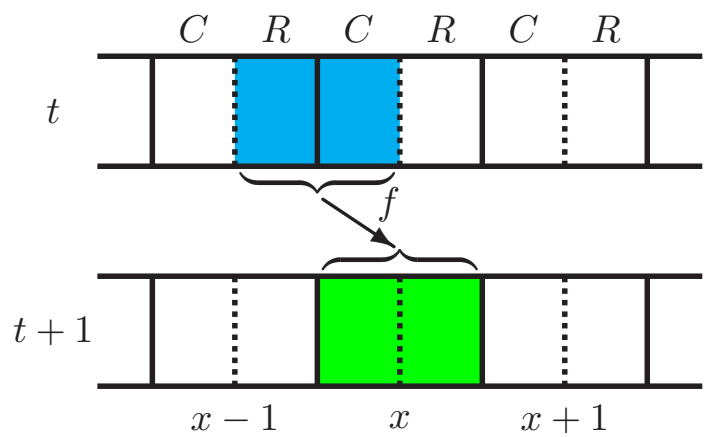

Figure 1: Cellular space of a one-dimensional 2-neighbor PCA, and its local function $f$. 


\section{Converting an RPCA into an RNCCA}

Lemma 2 For any given one-dimensional 2-neigbor $R P C A P=(\mathbb{Z},(C, R),(0,-1), f)$, we can construct a one-dimensional 4-neigbor RNCCA A that simulates $P$ and has $4|C| \cdot|R|$ states.

Proof. An RNCCA $A$ that simulates $P$ is given as follows.

$$
A=(\mathbb{Z}, \tilde{Q},(-2,-1,0,1), \tilde{f}, 0),
$$

where $\tilde{Q}=\{0,1, \ldots, 4|C| \cdot|R|-1\}$.

We need some preparations to define $\tilde{f}$. Let $\hat{C}, \check{C}, \tilde{C}, \hat{R}, \check{R}$, and $\tilde{R}$ be as follows.

$$
\begin{aligned}
& \hat{C}=\{2 k|R||k=0,1, \ldots,| C \mid-1\} \\
& \check{C}=\{2(k+|C|)|R||k=0,1, \ldots,| C \mid-1\} \\
& \tilde{C}=\grave{C} \cup \check{C} \\
& \hat{R}=\{k|k=0,1, \ldots,| R \mid-1\} \\
& \check{R}=\{k+|R||k=0,1, \ldots,| R \mid-1\} \\
& \tilde{R}=\hat{R} \cup \check{R}
\end{aligned}
$$

Each element $\tilde{c} \in \tilde{C}(\tilde{r} \in \tilde{R}$, respectively) is called a heavy (light) particle, which is a stationary (rightmoving) particle in $A$ as explained later. The number $\tilde{c}$ ( $\tilde{r}$, respectively) itself can be regarded as the mass of the heavy (light) particle. (Note that readers may think it strange that the particle of mass 0 is both heavy and light. Though the mass 0 could be considered as non-existence of a particle, we employ here the above interpretation for simplicity.) Clearly every element $\tilde{q} \in \tilde{Q}$ is uniquely decomposed into a heavy particle and a light particle, and thus the following holds.

$$
\begin{aligned}
& \forall \tilde{q} \in \tilde{Q}, \exists \tilde{c} \in \tilde{C}, \exists \tilde{r} \in \tilde{R}(\tilde{q}=\tilde{c}+\tilde{r}) \\
& \forall \tilde{c}_{1}, \tilde{c}_{2} \in \tilde{C}, \forall \tilde{r}_{1}, \tilde{r}_{2} \in \tilde{R}\left(\tilde{c}_{1}+\tilde{r}_{1}=\tilde{c}_{2}+\tilde{r}_{2} \Rightarrow \tilde{c}_{1}=\tilde{c}_{2} \wedge \tilde{r}_{1}=\tilde{r}_{2}\right)
\end{aligned}
$$

We can thus regard each cell of $A$ has a heavy particle and a light particle. We define the following functions $\tilde{p}_{C}: \tilde{Q} \rightarrow \tilde{C}$, and $\tilde{p}_{R}: \tilde{Q} \rightarrow \tilde{R}$, which give a heavy particle, and a light particle associated with a given $\tilde{q} \in \tilde{Q}$.

$$
\begin{aligned}
& \forall \tilde{q} \in \tilde{Q}, \forall \tilde{c} \in \tilde{C}\left(\tilde{p}_{C}(\tilde{q})=\tilde{c} \Leftrightarrow \exists \tilde{r} \in \tilde{R}(\tilde{q}=\tilde{c}+\tilde{r})\right) \\
& \forall \tilde{q} \in \tilde{Q}, \forall \tilde{r} \in \tilde{R}\left(\tilde{p}_{R}(\tilde{q})=\tilde{r} \Leftrightarrow \exists \tilde{c} \in \tilde{C}(\tilde{q}=\tilde{c}+\tilde{r})\right)
\end{aligned}
$$

A pair of heavy particles $(\hat{c}, \check{c}) \in \hat{C} \times \check{C}$ (light particles $(\hat{r}, \check{r}) \in \hat{R} \times \check{R}$, respectively) is called a complementary pair, if $\hat{c}+\check{c}=2(2|C|-1)|R|(\hat{r}+\check{r}=2|R|-1)$. In the following, a complementary pair of heavy (light, respectively) particles is used to simulate a state in $C(R)$. A pair of states $\left(\tilde{q}_{1}, \tilde{q}_{2}\right) \in \tilde{Q}^{2}$ is called balanced with respect to heavy (light, respectively) particles if $\left(\tilde{p}_{C}\left(\tilde{q}_{1}\right), \tilde{p}_{C}\left(\tilde{q}_{2}\right)\right)\left(\left(\tilde{p}_{R}\left(\tilde{q}_{1}\right), \tilde{p}_{R}\left(\tilde{q}_{2}\right)\right)\right)$ is a complementary pair. The set of all balanced pairs of states $\left(\tilde{q}_{1}, \tilde{q}_{2}\right)$ with respect to heavy (light, respectively) particles is denoted by $B_{C}\left(B_{R}\right)$, i.e.,

$$
\begin{aligned}
B_{C}=\left\{\left(\tilde{q}_{1}, \tilde{q}_{2}\right) \in \tilde{Q}^{2} \mid\right. & \left(\tilde{p}_{C}\left(\tilde{q}_{1}\right), \tilde{p}_{C}\left(\tilde{q}_{2}\right)\right) \in \hat{C} \times \check{C} \\
& \left.\wedge \tilde{p}_{C}\left(\tilde{q}_{1}\right)+\tilde{p}_{C}\left(\tilde{q}_{2}\right)=2(2|C|-1)|R|\right\}, \\
B_{R}=\left\{\left(\tilde{q}_{1}, \tilde{q}_{2}\right) \in \tilde{Q}^{2} \mid\right. & \left(\tilde{p}_{R}\left(\tilde{q}_{1}\right), \tilde{p}_{R}\left(\tilde{q}_{2}\right)\right) \in \hat{R} \times \check{R} \\
& \left.\wedge \tilde{p}_{R}\left(\tilde{q}_{1}\right)+\tilde{p}_{R}\left(\tilde{q}_{2}\right)=2|R|-1\right\} .
\end{aligned}
$$

It is easy to see that, for any $\tilde{\alpha} \in \operatorname{Conf}(\tilde{Q})$ and $x \in \mathbb{Z}$, the following relations hold.

$$
\begin{aligned}
& (\tilde{\alpha}(x), \tilde{\alpha}(x+1)) \in B_{C} \Rightarrow(\tilde{\alpha}(x-1), \tilde{\alpha}(x)) \notin B_{C} \wedge(\tilde{\alpha}(x+1), \tilde{\alpha}(x+2)) \notin B_{C} \\
& (\tilde{\alpha}(x), \tilde{\alpha}(x+1)) \in B_{R} \Rightarrow(\tilde{\alpha}(x-1), \tilde{\alpha}(x)) \notin B_{R} \wedge(\tilde{\alpha}(x+1), \tilde{\alpha}(x+2)) \notin B_{R}
\end{aligned}
$$


We now choose bijections $\hat{\varphi}_{C}: C \rightarrow \hat{C}$, and $\hat{\varphi}_{R}: R \rightarrow \hat{R}$ arbitrarily, and fix them hereafter. Then define the bijections $\check{\varphi}_{C}: C \rightarrow \check{C}, \breve{\varphi}_{R}: R \rightarrow \check{R}, \hat{\varphi}: C \times R \rightarrow \hat{Q}$, and $\check{\varphi}: C \times R \rightarrow \check{Q}$ as follows, where $\hat{Q}=\{\hat{c}+\hat{r} \mid \hat{c} \in \hat{C}, \hat{r} \in \hat{R}\}$, and $\check{Q}=\{\check{c}+\check{r} \mid \check{c} \in \check{C}, \check{r} \in \check{R}\}$.

$$
\begin{aligned}
& \forall c \in C\left(\check{\varphi}_{C}(c)=2(2|C|-1)|R|-\hat{\varphi}_{C}(c)\right) \\
& \forall r \in R\left(\check{\varphi}_{R}(r)=2|R|-1-\hat{\varphi}_{R}(r)\right) \\
& \forall c \in C, \forall r \in R\left(\hat{\varphi}(c, r)=\hat{\varphi}_{C}(c)+\hat{\varphi}_{R}(r)\right) \\
& \forall c \in C, \forall r \in R\left(\breve{\varphi}(c, r)=\breve{\varphi}_{C}(c)+\breve{\varphi}_{R}(r)\right)
\end{aligned}
$$

Now, $\tilde{f}: \tilde{Q}^{4} \rightarrow \tilde{Q}$ is defined as follows.

$\tilde{f}\left(\tilde{q}_{-2}, \tilde{q}_{-1}, \tilde{q}_{0}, \tilde{q}_{1}\right)= \begin{cases}\hat{\varphi}\left(f\left(\hat{\varphi}_{C}^{-1}\left(\tilde{p}_{C}\left(\tilde{q}_{0}\right)\right), \hat{\varphi}_{R}^{-1}\left(\tilde{p}_{R}\left(\tilde{q}_{-1}\right)\right)\right)\right) & \text { if }\left(\tilde{q}_{-1}, \tilde{q}_{0}\right) \in B_{R} \wedge\left(\tilde{q}_{0}, \tilde{q}_{1}\right) \in B_{C} \\ \check{\varphi}\left(f\left(\hat{\varphi}_{C}^{-1}\left(\tilde{p}_{C}\left(\tilde{q}_{-1}\right)\right), \hat{\varphi}_{R}^{-1}\left(\tilde{p}_{R}\left(\tilde{q}_{-2}\right)\right)\right)\right) & \text { if }\left(\tilde{q}_{-2}, \tilde{q}_{-1}\right) \in B_{R} \wedge\left(\tilde{q}_{-1}, \tilde{q}_{0}\right) \in B_{C} \\ \tilde{p}_{C}\left(\tilde{q}_{0}\right)+\tilde{p}_{R}\left(\tilde{q}_{-1}\right) & \text { elsewhere }\end{cases}$

Let $\tilde{F}$ be the global function induced by $\tilde{f}$. For any configuration $\tilde{\alpha} \in \operatorname{Conf}(\tilde{Q})$, and for any $y \in \mathbb{Z}$, the value $\tilde{F}(\tilde{\alpha})(y)$ is as follows. If $(\tilde{\alpha}(y-1), \tilde{\alpha}(y)) \in B_{R} \wedge(\tilde{\alpha}(y), \tilde{\alpha}(y+1)) \in B_{C}$, then

$$
\begin{aligned}
\tilde{F}(\tilde{\alpha})(y) & =\hat{\varphi}\left(f\left(\hat{\varphi}_{C}^{-1}\left(\tilde{p}_{C}(\tilde{\alpha}(y))\right), \hat{\varphi}_{R}^{-1}\left(\tilde{p}_{R}(\tilde{\alpha}(y-1))\right)\right)\right), \\
\tilde{F}(\tilde{\alpha})(y+1) & =\check{\varphi}\left(f\left(\hat{\varphi}_{C}^{-1}\left(\tilde{p}_{C}(\tilde{\alpha}(y))\right), \hat{\varphi}_{R}^{-1}\left(\tilde{p}_{R}(\tilde{\alpha}(y-1))\right)\right)\right) .
\end{aligned}
$$

It means the complementary pairs $\left(\tilde{p}_{R}(\tilde{\alpha}(y-1)), \tilde{p}_{R}(\tilde{\alpha}(y))\right)$ and $\left(\tilde{p}_{C}(\tilde{\alpha}(y)), \tilde{p}_{C}(\tilde{\alpha}(y+1))\right)$ interact each other, and the state transition of the RPCA $P$ is simulated. Thus, the new complementary pair of heavy particles $\left(\tilde{p}_{C}(\tilde{F}(\tilde{\alpha})(y)), \tilde{p}_{C}(\tilde{F}(\tilde{\alpha})(y+1))\right)$ is created at the same position as before, while the pair of light particles $\left(\tilde{p}_{R}(\tilde{F}(\tilde{\alpha})(y)), \tilde{p}_{R}(\tilde{F}(\tilde{\alpha})(y+1))\right)$ appears at the position shifted rightward by one cell. On the other hand, if $\left.\neg\left((\tilde{\alpha}(y-2), \tilde{\alpha}(y-1)) \in B_{R} \wedge(\tilde{\alpha}(y-1), \tilde{\alpha}(y)) \in B_{C}\right)\right) \wedge \neg((\tilde{\alpha}(y-1), \tilde{\alpha}(y)) \in$ $\left.\left.B_{R} \wedge(\tilde{\alpha}(y), \tilde{\alpha}(y+1)) \in B_{C}\right)\right)$, then

$$
\tilde{F}(\tilde{\alpha})(y)=\tilde{p}_{C}(\tilde{\alpha}(y))+\tilde{p}_{R}(\tilde{\alpha}(y-1)) .
$$

The above means the light particle $\tilde{p}_{R}(\tilde{\alpha}(y-1))$ simply moves rightward without interacting with the stationary heavy particle $\tilde{p}_{C}(\tilde{\alpha}(y))$. From (2)-(4), it is easy to see that the following holds for all $x \in \mathbb{Z}$.

$$
\begin{array}{lll}
(\tilde{\alpha}(x), \tilde{\alpha}(x+1)) \in B_{C} & \Leftrightarrow & (\tilde{F}(\tilde{\alpha})(x), \tilde{F}(\tilde{\alpha})(x+1)) \in B_{C} \\
(\tilde{\alpha}(x), \tilde{\alpha}(x+1)) \in B_{R} & \Leftrightarrow & (\tilde{F}(\tilde{\alpha})(x+1), \tilde{F}(\tilde{\alpha})(x+2)) \in B_{R}
\end{array}
$$

First, we show that $A$ can simulate $P$ correctly as described below. After that, we will show $A$ is an RNCCA. We define a mapping $\tilde{\tau}: \operatorname{Conf}(C \times R) \rightarrow \operatorname{Conf}(\tilde{Q})$ as follows, where $\alpha \in \operatorname{Conf}(C \times R)$ and $x \in \mathbb{Z}$.

$$
\begin{aligned}
\tilde{\tau}(\alpha)(2 x) & =\hat{\varphi}(\alpha(x)) \\
\tilde{\tau}(\alpha)(2 x+1) & =\check{\varphi}(\alpha(x))
\end{aligned}
$$

The configuration $\alpha$ of $P$ is thus represented by $\tilde{\tau}(\alpha)$ of $A$ (see Fig. 2). We can see $\left(\tilde{p}_{C}(\tilde{\tau}(\alpha)(2 x))\right.$, $\left.\tilde{p}_{C}(\tilde{\tau}(\alpha)(2 x+1))\right) \in B_{C}$. However, $\left(\tilde{p}_{R}(\tilde{\tau}(\alpha)(2 x-1)), \tilde{p}_{R}(\tilde{\tau}(\alpha)(2 x))\right) \notin B_{R}$ for any $x \in \mathbb{Z}$. Therefore, by the equation (4) we have the following.

$$
\begin{aligned}
\tilde{F}(\tilde{\tau}(\alpha))(2 x) & =\tilde{p}_{C}(\hat{\varphi}(\alpha(x)))+\tilde{p}_{R}(\check{\varphi}(\alpha(x-1))) \\
\tilde{F}(\tilde{\tau}(\alpha))(2 x+1) & =\tilde{p}_{C}(\check{\varphi}(\alpha(x)))+\tilde{p}_{R}(\hat{\varphi}(\alpha(x)))
\end{aligned}
$$


By above, we can observe $\left(\tilde{p}_{C}(\tilde{F}(\tilde{\tau}(\alpha))(2 x)), \tilde{p}_{C}(\tilde{F}(\tilde{\tau}(\alpha))(2 x+1))\right) \in B_{C}$ and $\left(\tilde{p}_{R}(\tilde{F}(\tilde{\tau}(\alpha))(2 x-1))\right.$, $\left.\tilde{p}_{R}(\tilde{F}(\tilde{\tau}(\alpha))(2 x))\right) \in B_{R}$, and thus the following holds by (2) and (3), where $p_{C}: C \times R \rightarrow C$ and $p_{R}$ : $C \times R \rightarrow R$ are projection functions, and $F$ is the global function of $P$.

$$
\begin{aligned}
\tilde{F}^{2}(\tilde{\tau}(\alpha))(2 x) & =\hat{\varphi}\left(f\left(\hat{\varphi}_{C}^{-1}\left(\tilde{p}_{C}(\hat{\varphi}(\alpha(x)))\right), \hat{\varphi}_{R}^{-1}\left(\tilde{p}_{R}(\hat{\varphi}(\alpha(x-1)))\right)\right)\right) \\
& =\hat{\varphi}\left(f\left(p_{C}(\alpha(x)), p_{R}(\alpha(x-1))\right)\right) \\
& =\hat{\varphi}(F(\alpha)(x)) \\
& =\tilde{\tau}(F(\alpha))(2 x) \\
\tilde{F}^{2}(\tilde{\tau}(\alpha))(2 x+1) & =\check{\varphi}\left(f\left(\hat{\varphi}_{C}^{-1}\left(\tilde{p}_{C}(\hat{\varphi}(\alpha(x)))\right), \hat{\varphi}_{R}^{-1}\left(\tilde{p}_{R}(\hat{\varphi}(\alpha(x-1)))\right)\right)\right) \\
& =\check{\varphi}\left(f\left(p_{C}(\alpha(x)), p_{R}(\alpha(x-1))\right)\right) \\
& =\check{\varphi}(F(\alpha)(x)) \\
& =\tilde{\tau}(F(\alpha))(2 x+1)
\end{aligned}
$$

Thus, each evolution step of a configuration of $P$ is correctly simulated by $A$ in two steps under the mapping $\tilde{\tau}$. Its simulation process is shown in Fig. 2

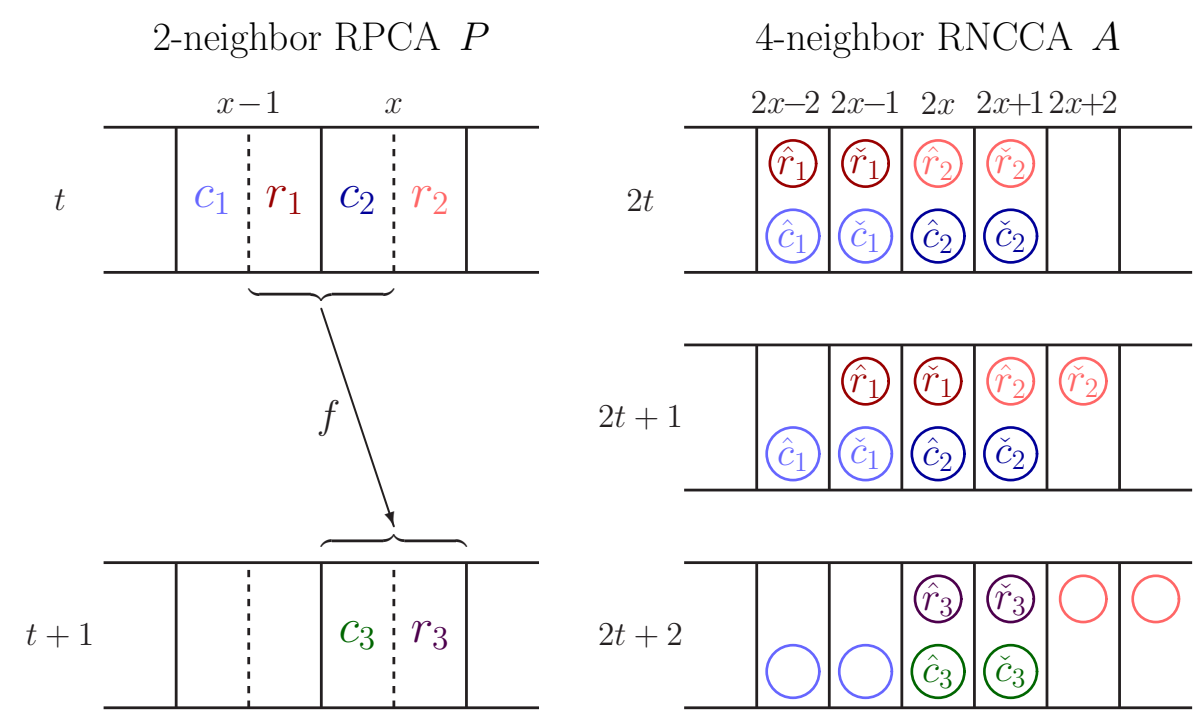

Figure 2: A simulation process of a 2-neighbor RPCA $P$ by a 4-neighbor RNCCA $A$. The configuration of $A$ at time $2 t$ is obtained from that of $P$ at time $t$ by the mapping $\tilde{\tau}$. Here, $\left(\hat{c}_{i}, \breve{c}_{i}\right)$ and $\left(\hat{r}_{i}, \breve{r}_{i}\right)(i=1,2,3)$ are complementary pairs, and thus $\hat{c}_{i}+\breve{c}_{i}=2(2|C|-1)|R|$ and $\hat{r}_{i}+\breve{r}_{i}=2|R|-1$.

Next, we show that $A$ is an NCCA. From the equation (1), we can see mass of a particle is transferred within a complementary pair, or simply shifted rightward, or does not change. Therefore, it is intuitively obvious that $A$ is an NCCA. But, here we show that $A$ has the finite-number-conserving property. First, 
from (2)-(4), we can derive the following.

$$
\begin{aligned}
& (\tilde{\alpha}(x), \tilde{\alpha}(x+1)) \in B_{C} \\
& \quad \Rightarrow \tilde{p}_{C}(\tilde{\alpha}(x))+\tilde{p}_{C}(\tilde{\alpha}(x+1))=\tilde{p}_{C}(\tilde{F}(\tilde{\alpha})(x))+\tilde{p}_{C}(\tilde{F}(\tilde{\alpha})(x+1)) \\
& (\tilde{\alpha}(x-1), \tilde{\alpha}(x)) \notin B_{C} \wedge(\tilde{\alpha}(x), \tilde{\alpha}(x+1)) \notin B_{C} \\
& \quad \Rightarrow \tilde{p}_{C}(\tilde{\alpha}(x))=\tilde{p}_{C}(\tilde{F}(\tilde{\alpha})(x)) \\
& (\tilde{\alpha}(x), \tilde{\alpha}(x+1)) \in B_{R} \\
& \quad \Rightarrow \tilde{p}_{R}(\tilde{\alpha}(x))+\tilde{p}_{R}(\tilde{\alpha}(x+1))=\tilde{p}_{R}(\tilde{F}(\tilde{\alpha})(x+1))+\tilde{p}_{R}(\tilde{F}(\tilde{\alpha})(x+2)) \\
& (\tilde{\alpha}(x-1), \tilde{\alpha}(x)) \notin B_{R} \wedge(\tilde{\alpha}(x), \tilde{\alpha}(x+1)) \notin B_{R} \\
& \quad \Rightarrow \tilde{p}_{R}(\tilde{\alpha}(x))=\tilde{p}_{R}(\tilde{F}(\tilde{\alpha})(x+1))
\end{aligned}
$$

By above, for each $n(=2,3, \ldots)$, there exist $n_{0}, n_{1}$ and $n_{2}$ such that $n_{i} \in\{n, n-1\}(i=0,1,2)$, and $n_{3} \in\{n-1, n-2\}$ that satisfy the following relations.

$$
\begin{aligned}
\sum_{x=-n_{0}}^{n_{1}} \tilde{p}_{C}(\tilde{\alpha}(x)) & =\sum_{x=-n_{0}}^{n_{1}} \tilde{p}_{C}(\tilde{F}(\tilde{\alpha})(x)) \\
\sum_{x=-n_{2}}^{n_{3}} \tilde{p}_{R}(\tilde{\alpha}(x)) & =\sum_{x=-n_{2}+1}^{n_{3}+1} \tilde{p}_{R}(\tilde{F}(\tilde{\alpha})(x))
\end{aligned}
$$

Let $\tilde{\alpha} \in \operatorname{Conf}_{\text {fin }}(\tilde{Q})$. Then, the following equation holds by (9) and (10).

$$
\forall \tilde{\alpha} \in \operatorname{Conf}(\tilde{Q}): \sum_{x \in \mathbb{Z}} \tilde{\alpha}(x)=\sum_{x \in \mathbb{Z}} \tilde{F}(\tilde{\alpha})(x)
$$

Therefore, $A$ is finite-number-conserving, and thus an NCCA.

Finally, we show $A$ is reversible. On the contrary we assume it is not. Thus, there are two configurations $\tilde{\alpha}_{1}, \tilde{\alpha}_{2} \in \operatorname{Conf}(\tilde{Q})$ such that $\tilde{\alpha}_{1} \neq \tilde{\alpha}_{2}$ and $\tilde{F}\left(\tilde{\alpha}_{1}\right)=\tilde{F}\left(\tilde{\alpha}_{2}\right)$. First, we note the following.

$$
\begin{aligned}
& \forall x \in \mathbb{Z}\left(\left(\tilde{\alpha}_{1}(x), \tilde{\alpha}_{1}(x+1)\right) \in B_{C} \Leftrightarrow\left(\tilde{\alpha}_{2}(x), \tilde{\alpha}_{2}(x+1)\right) \in B_{C}\right) \\
& \forall x \in \mathbb{Z}\left(\left(\tilde{\alpha}_{1}(x), \tilde{\alpha}_{1}(x+1)\right) \in B_{R} \Leftrightarrow\left(\tilde{\alpha}_{2}(x), \tilde{\alpha}_{2}(x+1)\right) \in B_{R}\right)
\end{aligned}
$$

If otherwise, $\tilde{F}\left(\tilde{\alpha}_{1}\right) \neq \tilde{F}\left(\tilde{\alpha}_{2}\right)$ holds by the relations (5) and (6), and it contradicts the assumption. Since $\tilde{\alpha}_{1} \neq \tilde{\alpha}_{2}$, there exists $x_{0} \in \mathbb{Z}$ such that $\tilde{p}_{C}\left(\tilde{\alpha}_{1}\left(x_{0}\right)\right) \neq \tilde{p}_{C}\left(\tilde{\alpha}_{2}\left(x_{0}\right)\right)$ or $\tilde{p}_{R}\left(\tilde{\alpha}_{1}\left(x_{0}\right)\right) \neq \tilde{p}_{R}\left(\tilde{\alpha}_{2}\left(x_{0}\right)\right)$. Here, we prove it only for the case $\tilde{p}_{C}\left(\tilde{\alpha}_{1}\left(x_{0}\right)\right) \neq \tilde{p}_{C}\left(\tilde{\alpha}_{2}\left(x_{0}\right)\right)$, since the case $\tilde{p}_{R}\left(\tilde{\alpha}_{1}\left(x_{0}\right)\right) \neq \tilde{p}_{R}\left(\tilde{\alpha}_{2}\left(x_{0}\right)\right)$ is similarly proved. There are three subcases:

(i) $\left(\tilde{\alpha}_{i}\left(x_{0}-1\right), \tilde{\alpha}_{i}\left(x_{0}\right)\right) \in B_{R} \wedge\left(\tilde{\alpha}_{i}\left(x_{0}\right), \tilde{\alpha}_{i}\left(x_{0}+1\right)\right) \in B_{C}(i=1,2)$,

(ii) $\left(\tilde{\alpha}_{i}\left(x_{0}-2\right), \tilde{\alpha}_{i}\left(x_{0}-1\right)\right) \in B_{R} \wedge\left(\tilde{\alpha}_{i}\left(x_{0}-1\right), \tilde{\alpha}_{i}\left(x_{0}\right)\right) \in B_{C}(i=1,2)$, and

(iii) Other than the cases (i) and (ii), i.e., $\neg\left(\left(\tilde{\alpha}_{i}\left(x_{0}-1\right), \tilde{\alpha}_{i}\left(x_{0}\right)\right) \in B_{R} \wedge\left(\tilde{\alpha}_{i}\left(x_{0}\right), \tilde{\alpha}_{i}\left(x_{0}+1\right)\right) \in B_{C}\right) \wedge$ $\neg\left(\left(\tilde{\alpha}_{i}\left(x_{0}-2\right), \tilde{\alpha}_{i}\left(x_{0}-1\right)\right) \in B_{R} \wedge\left(\tilde{\alpha}_{i}\left(x_{0}-1\right), \tilde{\alpha}_{i}\left(x_{0}\right)\right) \in B_{C}\right)(i=1,2)$.

The case (i): By (2), the following relations hold.

$$
\begin{aligned}
\tilde{F}\left(\tilde{\alpha}_{1}\right)\left(x_{0}\right) & =\hat{\varphi}\left(f\left(\hat{\varphi}_{C}^{-1}\left(\tilde{p}_{C}\left(\tilde{\alpha}_{1}\left(x_{0}\right)\right)\right), \hat{\varphi}_{R}^{-1}\left(\tilde{p}_{R}\left(\tilde{\alpha}_{1}\left(x_{0}-1\right)\right)\right)\right)\right) \\
\tilde{F}\left(\tilde{\alpha}_{2}\right)\left(x_{0}\right) & =\hat{\varphi}\left(f\left(\hat{\varphi}_{C}^{-1}\left(\tilde{p}_{C}\left(\tilde{\alpha}_{2}\left(x_{0}\right)\right)\right), \hat{\varphi}_{R}^{-1}\left(\tilde{p}_{R}\left(\tilde{\alpha}_{2}\left(x_{0}-1\right)\right)\right)\right)\right)
\end{aligned}
$$


From the facts $\tilde{p}_{C}\left(\tilde{\alpha}_{1}\left(x_{0}\right)\right) \neq \tilde{p}_{C}\left(\tilde{\alpha}_{2}\left(x_{0}\right)\right), \varphi, \varphi_{C}$ and $\varphi_{R}$ are bijections, and $f$ is an injection (because $P$ is a reversible PCA), $\tilde{F}\left(\tilde{\alpha}_{1}\right)\left(x_{0}\right) \neq \tilde{F}\left(\tilde{\alpha}_{2}\right)\left(x_{0}\right)$ follows. This contradicts the assumption. The case (ii): Since it is similar to the case (i), we omit the proof. The case (iii): By (4), the following relations hold.

$$
\begin{aligned}
& \tilde{F}\left(\tilde{\alpha}_{1}\right)\left(x_{0}\right)=\tilde{p}_{C}\left(\tilde{\alpha}_{1}\left(x_{0}\right)\right)+\tilde{p}_{R}\left(\tilde{\alpha}_{1}\left(x_{0}-1\right)\right) \\
& \tilde{F}\left(\tilde{\alpha}_{2}\right)\left(x_{0}\right)=\tilde{p}_{C}\left(\tilde{\alpha}_{2}\left(x_{0}\right)\right)+\tilde{p}_{R}\left(\tilde{\alpha}_{2}\left(x_{0}-1\right)\right)
\end{aligned}
$$

Again $\tilde{F}\left(\tilde{\alpha}_{1}\right)\left(x_{0}\right) \neq \tilde{F}\left(\tilde{\alpha}_{2}\right)\left(x_{0}\right)$, because $\tilde{p}_{C}\left(\tilde{\alpha}_{1}\left(x_{0}\right)\right) \neq \tilde{p}_{C}\left(\tilde{\alpha}_{2}\left(x_{0}\right)\right)$, and this contradicts the assumption. By above, we can conclude that $A$ is a reversible NCCA. This completes the proof.

It has been shown that there is a universal one-dimensional 2-neighbor 24-state RPCA [12]. This RPCA can simulate Any cyclic tag system proposed by Cook [2] can be simulated by this RPCA with infinite but ultimately-periodic configurations.

Proposition 1 [12] There is a computationally universal one-dimensional 2-neighbor 24-state RPCA.

From Lemma 2 and Proposition 1 the next theorem is derived.

Theorem 1 There is a computationally universal one-dimensional 4-neighbor 96-state RNCCA.

In [12], it is shown that there is a 2-neighbor RPCA that directly simulates a given reversible Turing machine. Therefore, we can also construct a 4-neighbor RNCCA that directly simulates a reversible Turing machine. In this case, the RNCCA has ultimately periodic infinite configurations, though the configuration of the simulated Turing machine is finite.

\section{Concluding remarks}

In this paper, we proved that any given 2-neighbor RPCA $P$ can be simulated by a 4-neighbor RNCCA $A$. Thus computation-universality of a 4-neighbor RNCCA is concluded in spite of the strong constraints of reversibility and the number-conserving property. When $A$ simulates $P$, a configuration $\alpha$ of $P$ is kept by $\tilde{\tau}(\alpha)$ of $A$ as shown in Fig. 2. But, there is no need to define $\tilde{\tau}$ as given in the equations (7) and (8). The simulation works well if we use, e.g., the following $\tilde{\tau}^{\prime}$, where each two-cell-block containing $\hat{\varphi}(\alpha(x))$ and $\breve{\varphi}(\alpha(x))$ is separated from the next block by $(k-2) 0$-state cells $(k=3,4, \ldots)$.

$$
\begin{aligned}
\tilde{\tau}^{\prime}(\alpha)(k x) & =\hat{\varphi}(\alpha(x)) \\
\tilde{\tau}^{\prime}(\alpha)(k x+1) & =\grave{\varphi}(\alpha(x)) \\
\tilde{\tau}^{\prime}(\alpha)(k x+i) & =0 \quad(i=2,3, \ldots, k-1)
\end{aligned}
$$

Furthermore, we can see that, even if the spacing between blocks (by 0-state cells) is non-uniform, the simulation process goes correctly (though state transition timing of the cells are also non-uniform).

On the other hand, it is an open problem whether a stronger result holds, i.e., whether there is a universal 3-neighbor (radius 1) RNCCA. It is also left for the future study to construct an intrinsically universal RNCCA.

Acknowledgement. This work was supported in part by JSPS Grant-in-Aid for Scientific Research (C) No. 21500015 and No. 24500017. 


\section{References}

[1] N. Boccara \& H. Fukś (2002): Number-conserving cellular automata rules. Fundamenta Informaticae 52, pp. 1-13.

[2] M. Cook (2004): Universality in elementary cellular automata. Complex Syst. 15, pp. 1-40.

[3] B. Durand, E. Formenti \& Z. Róka (2003): Number-conserving cellular automata I: decidability. Theoret. Comput. Sci. 299, pp. 523-535, doi 10.1016/S0304-3975(02)00534-0

[4] E. Formenti \& A. Grange (2003): Number conserving cellular automata II: dynamics. Theoret. Comput. Sci. 304, pp. 269-290, doi:10.1016/S0304-3975(03)00134-8.

[5] H. Fukś \& K. Sullivan (2007): Enumeration of number-conserving cellular automata rules with two inputs. J. Cell. Autom. 2, pp. 141-148.

[6] F. García-Ramos (2012): Product decomposition for surjective 2-block NCCA. In: DMTCS Proc. on AUTOMATA 2011, pp. 147-158.

[7] T. Hattori \& S. Takesue (1991): Additive conserved quantities in discrete-time lattice dynamical systems. Physica D 49, pp. 295-322, doi:10.1016/0167-2789(91)90150-8.

[8] K. Imai, B. Martin \& R. Saito (2012): On radius 1 nontrivial reversible and number-conserving cellular automata. In: Proc. RC 2012, pp. 54-60.

[9] J. Kari \& S. Taati (2008): Particle displacement representation for conservative laws in two-dimensional cellular automata. In: Proc. JAC 2008, pp. 65-73.

[10] A. Moreira (2003): Universality and decidability of number-conserving cellular automata. Theoret. Comput. Sci. 292, pp. 711-721, doi 10.1016/S0304-3975(02)00065-8

[11] K. Morita (2008): Reversible computing and cellular automata - A survey. Theoret. Comput. Sci. 395, pp. 101-131, doi:10.1016/j.tcs.2008.01.041

[12] K. Morita (2011): Simulating reversible Turing machines and cyclic tag systems by one-dimensional reversible cellular automata. Theoret. Comput. Sci., pp. 3856-3865, doi $10.1016 /$ j.tcs.2011.02.022

[13] K. Morita \& M. Harao (1989): Computation universality of one-dimensional reversible (injective) cellular automata. Trans. IEICE Japan E72, pp. 758-762.

[14] K. Morita \& K. Imai (2001): Number-conserving reversible cellular automata and their computationuniversality. Theoret. Informat. Appl. 35, pp. 239-258, doi 10.1051/ita:2001118

[15] A. Schranko \& P.P.B. de Oliveira (2010): Derivation and representation of one-dimensional, reversible, number-conserving cellular automata rules. J. Cell. Autom. 6, pp. 77-89. 